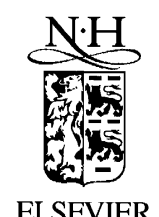

ELSEVIER

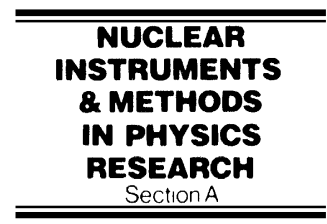

www.elsevier.com/locate/nima

\title{
RPC ageing studies
}

\author{
G. Aielli*, P. Camarri, R. Cardarelli, R. de Asmundis, A. Di Ciaccio, \\ L. Di Stante, B. Liberti, A. Paoloni, E. Pastori, R. Santonico \\ Dipartimento di Fisica dell'Università di Roma "Tor Vergata", INFN Sezione di Roma 2, \\ Via della Ricerca Scientifica 1, 00133 Roma, Italy
}

\begin{abstract}
A 15-month ageing test of an ATLAS module " 0 " RPC has been performed at the GIF X5 CERN irradiation facility. The aged RPC performances largely fulfilled the ATLAS requirements. However, the test showed a significant reduction of the RPC maximum rate capability, from almost $2 \mathrm{kHz} \mathrm{cm}^{-2}$ to a few hundred $\mathrm{Hz} \mathrm{cm}^{-2}$, due to the total charge integrated during the test. The reasons for this ageing phenomenon are found to be related to an increase of the total electrode plates resistance as it was already pointed out in previous tests on smaller prototypes. This is found to be not only due to a possible increase of the plate resistivity but mainly to a degradation of the graphite coating. (C) 2002 Elsevier Science B.V. All rights reserved.
\end{abstract}

PACS: $29.40 . \mathrm{Cs}$

Keywords: RPC; Ageing; High rate; Front-end electronics; ATLAS

\section{The ageing problem in the RPC}

Several tests carried out on small size RPCs $[1,6]$, working under intense irradiation of gamma sources, showed that the dominant ageing effect was the progressive decrease of the rate capability. This effect was found to be due to a relevant increase of the electrodes resistance which is related to the integral of the detector working current, flowed across the electrode plates.

In order to perform a test equivalent to 10 ATLAS Years (AY) operation, we followed the criteria exposed below. The expected background counting rate for the RPCs in the ATLAS barrel

\footnotetext{
*Corresponding author. Tel.: + 39-6-7259; fax: + 39-06-2023507.

E-mail address: aielli@roma2.infn.it (G. Aielli).
}

region of $\sim 10 \mathrm{~Hz} \mathrm{~cm}^{-2}$ [1] was multiplied by a safety factor of 10 . We assumed, therefore, a baseline of $100 \mathrm{~Hz} \mathrm{~cm}^{-2}$, corresponding to a total counting of $10^{10} \mathrm{~cm}^{-2}$ for $10 \mathrm{AY}$, both for performance and ageing calculations. The average charge per event delivered in the gas at the working point is $\sim 30 \mathrm{pC}$, with the present layout. This corresponds to a total charge of $0.3 \mathrm{C} \mathrm{cm}^{-2}$ through the resistive plates.

\section{Experimental set-up and data taking}

An ageing test on a large size RPC, $176.7 \times$ $80.6 \mathrm{~cm}^{2}$ sensitive surface, was performed at the GIF facility of CERN. The GIF is equipped with a ${ }^{137} \mathrm{Cs} 740 \mathrm{GBq}$ photon source and is served by the $\mathrm{X} 5$ beam for test purposes. The photon flux on a 
large area detector is made uniform by a properly shaped lead filter and can be attenuated by further filters; the reduction factors used for this test are 2, 5, 10 and 20. A simulation of the experimental area [5] predicts a direct photon flux at full intensity of $4 \times 10^{5} \mathrm{~s}^{-1} \mathrm{~cm}^{-2}$, at a distance of $220 \mathrm{~cm}$ chosen for this test. The total flux, including the scattered photons is $6.2 \times 10^{5} \mathrm{~s}^{-1} \mathrm{~cm}^{-2}$. At full intensity, the overall counting rate was $\sim 1.6 \mathrm{kHz} \mathrm{cm}^{-2}$ at the plateau knee; this gives a sensitivity of $0.3 \%$ to the GIF photons.

We tested a single gap RPC made with Bakelite plates of $2 \times 10^{10} \Omega \mathrm{cm}$ bulk resistivity. The plates surfaces facing the gas were coated with a few micron thick layer of polymerized linseed oil. The readout was performed on both views by two orthogonal systems of terminated strip lines of $3.1 \mathrm{~cm}$ pitch. The strips were connected to 8channel boards, equipped with the GaAs FrontEnd (FE) electronics. The FE output signals were sent to a receiver through $14 \mathrm{~m}$ long flat cables. They were subsequently acquired by a multi-hit TDC, after being transmitted in the counting room by a $35 \mathrm{~m}$ long twisted pair cables.

The RPC was filled with the baseline gas mixture for ATLAS, suitable for safe operation in saturated avalanche mode [2]: $96.7 \%$ of tetrafluorethane, $3 \%$ of iso-butane and $0.3 \%$ of $\mathrm{SF}_{6}$.

The test required about 15 months of chamber operation in order to integrate the wanted charge amount, since the source could not always be used at full intensity. We monitored the total current, the counting rate, the environment parameters and the gas composition. Periodically, we measured the currents and the counting rates for different source intensities and/or the detector performance with the $100 \mathrm{GeV}$ muon beam or with cosmic rays when the beam was not available.

\section{Experimental results and discussion}

The detector performance at $3 \mathrm{AY}\left(0.1 \mathrm{C} \mathrm{cm}^{-2}\right)$ was measured using the $\mathrm{X} 5100 \mathrm{GeV}$ muon beam, triggered by three scintillators on a $10 \times 10 \mathrm{~cm}^{2}$ area. The efficiency plateau, shown in Fig. 1 for closed source and for half and full intensity [3], indicates that the detector could properly work

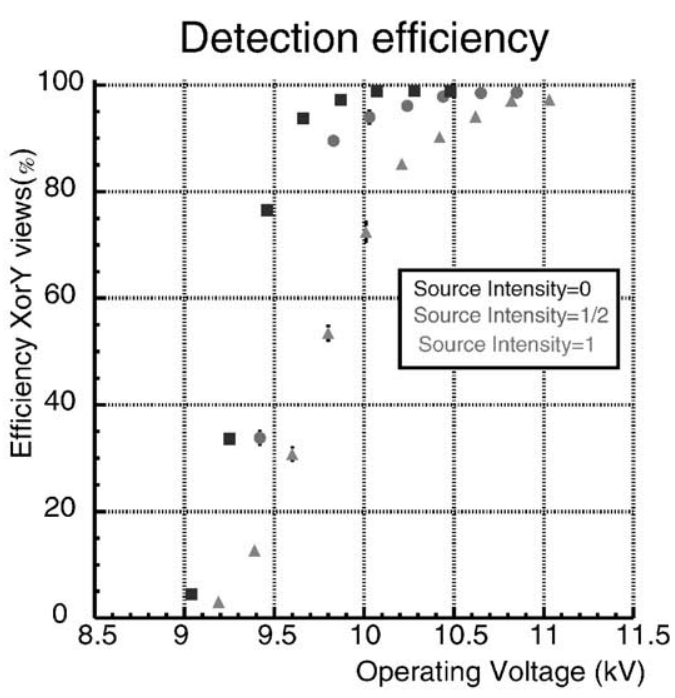

Fig. 1. Efficiency vs. applied voltage at $3 \mathrm{AY}$.

under a rate of about $1.6 \mathrm{kHz} \mathrm{cm}{ }^{-2}$. The efficiency was computed by selecting only the clusters firing inside the triggered area within a fixed time window of $10 \mathrm{~ns}$. The time resolution was $1.3 \mathrm{~ns}$ at full source. The cluster size was 1.2 at the efficiency knee and 1.4 for an overvoltage of $700 \mathrm{~V}$.

The performance measurement was repeated at $12 \mathrm{AY}$, during the winter beam stop, using cosmic rays triggered by a telescope of three $10 \times 40 \mathrm{~cm}^{2}$ scintillators $3 \mathrm{~cm}$ thick. The telescope was mounted onto the detector, with its axis at $35^{\circ}$ with respect to the vertical direction. The efficiency was measured by the coincidence of the trigger signal with the chamber total OR. The trigger rate was unaffected by the photon flux even for full intensity. The detection efficiency vs. voltage (Fig. 2) shows that the detector is still efficient at a rate of $300 \mathrm{~Hz} \mathrm{~cm}^{-2}$. The cluster size and the time resolution are compatible with the values measured at the beginning of the test for the same efficiency.

Fig. 3 shows the total current for different source intensities as a function of the applied voltage after $12 \mathrm{AY}$. We can clearly see that above $8 \mathrm{kV}$, the operating current scales as expected with the source intensity while for lower voltages, the bias current appears to be very small (e.g. $<1 \mu \mathrm{A}$ at $7 \mathrm{kV}$ ). The closed source counting rate at the efficiency knee was of $\sim 1 \mathrm{~Hz} \mathrm{~cm}{ }^{-2}$. Both values 


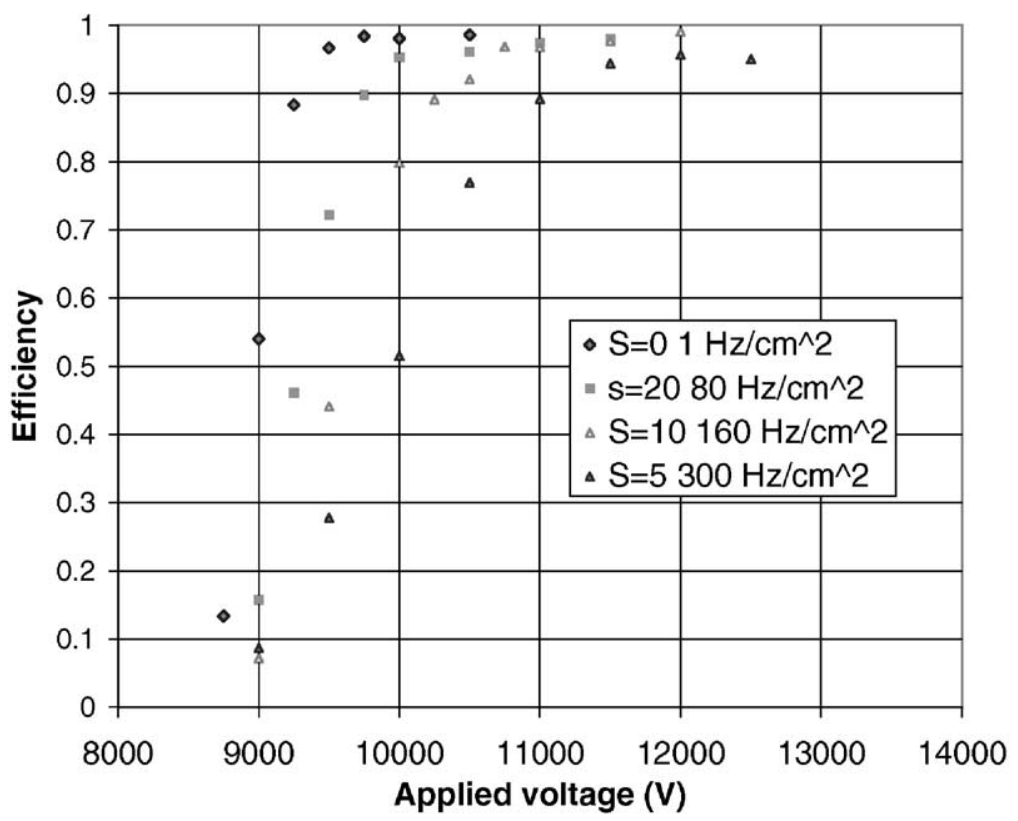

Fig. 2. Efficiency vs. applied voltage at $12 \mathrm{AY}$.

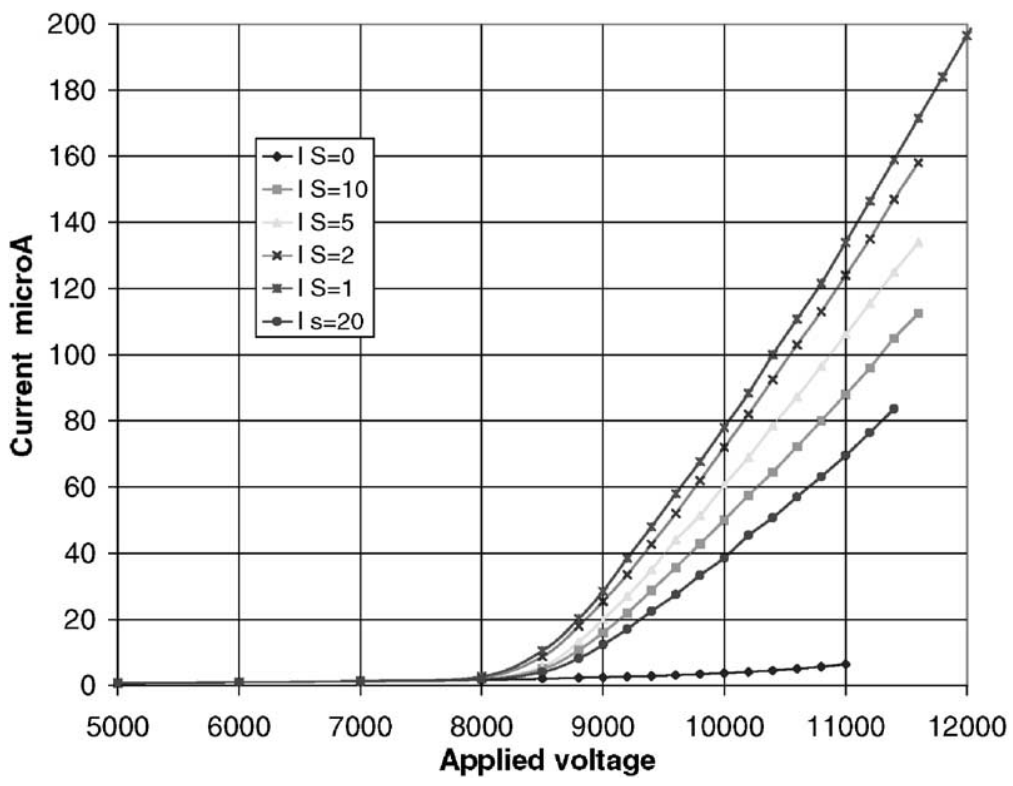

Fig. 3. Total operating current vs. applied voltage at $12 \mathrm{AY}$.

are compatible with the one measured at the beginning of the test.

The experimental results presented above can be interpreted according to the following ideas. If we consider the voltage drop across the resistive plates for a uniformly irradiated detector, it is a good approximation to consider $V_{\text {gas }}=V-I R_{\mathrm{b}}$ as the effective average voltage applied to the gas. $I$ and 
$R_{\mathrm{b}}$ are, respectively, the measured total current and RPCs electrode resistance.

If the detection efficiency is plotted vs. $V_{\text {gas }}$ (Fig. 4) instead of $V$ (Fig. 2), the points corresponding to different source intensities overlap on the same curve, meaning that the working point is only dependent on $V_{\text {gas }}$. Furthermore, this curve overlaps the closed source efficiency plateau after 3 AY (Fig. 1), as expected.

This confirms that the observed ageing effect can be described in terms of an increase of the operating voltage, depending on the actual ionization rate, due to the voltage drop on the increased electrode resistance.

\section{RPCs electrode behaviour}

Fig. 5 plots the total electrode resistance of the tested RPC vs. the integrated ageing in AY. The measurement was performed with a non-destructive method, based on the use of $V_{\text {gas }}$, already presented in Ref. [4]. The resistance shows a moderate increase up to about $10 \mathrm{AY}$ and it becomes steeper beyond.
In order to understand the basic phenomena producing the observed effects, a parallel ageing test was carried out on several $10 \times 10 \mathrm{~cm}^{2}$ Bakelite plates, $2 \mathrm{~mm}$ thick, with graphite coated faces. The effect of the current flowing across the detector was reproduced with a voltage of $400 \mathrm{~V}$ applied to the graphite electrodes, while the current, the temperature and the air humidity were monitored.

The electrode plate resistivity is shown in Fig. 6 vs. the integrated charge. The black curve refers to a test with the same graphite coating used in the case of the chamber tested at GIF. Short after integrating $0.4 \mathrm{C} \mathrm{cm}^{-2}$, the plate resistance (here interpreted as bulk resistivity) shows an abrupt increase. A dashed horizontal line indicates the maximum resistivity reached in the GIF test.

On the other side, measurements carried out during the plate ageing test, showed a selective increase of the anodic graphite layer resistivity from the initial value of $100 \mathrm{k} \Omega$ to $1 \mathrm{M} \Omega$, above $0.3 \mathrm{C} \mathrm{cm}^{-2}$, while the cathode remained almost unchanged. A more dramatic increase (up to $20 \mathrm{M} \Omega$ ) was observed above this limit. This test shows, therefore, a clear ageing effect due to the anodic graphite degradation.

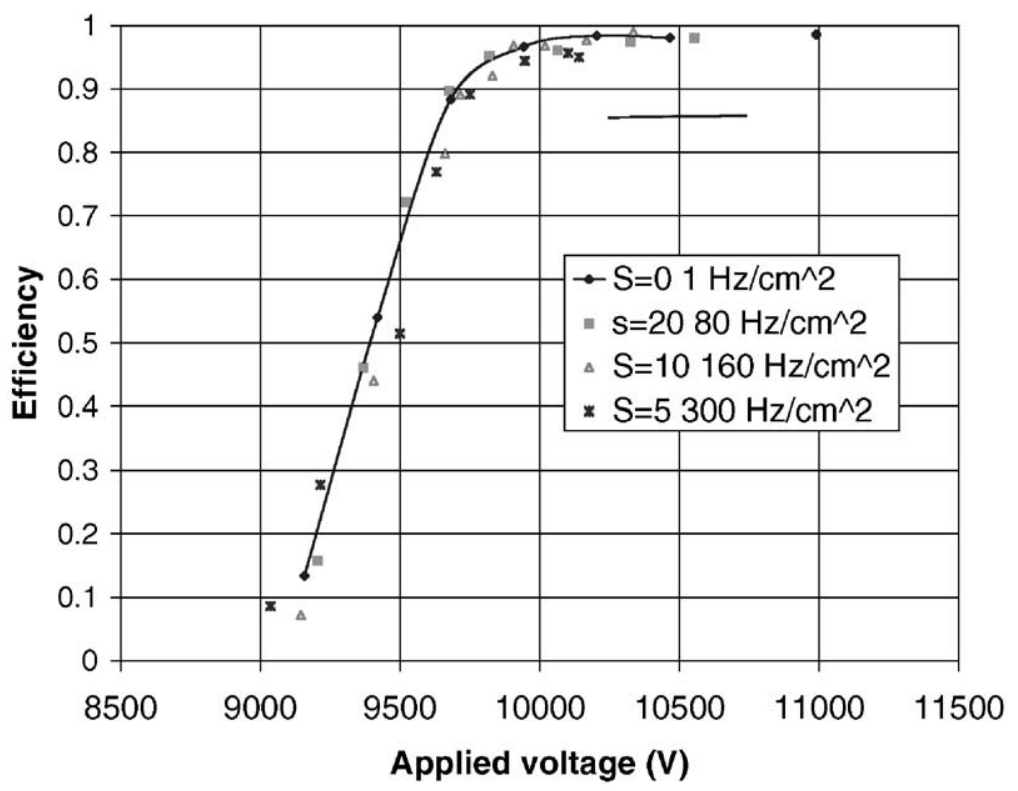

Fig. 4. Efficiency vs. $V_{\text {gas }}$ at $12 \mathrm{AY}$. 


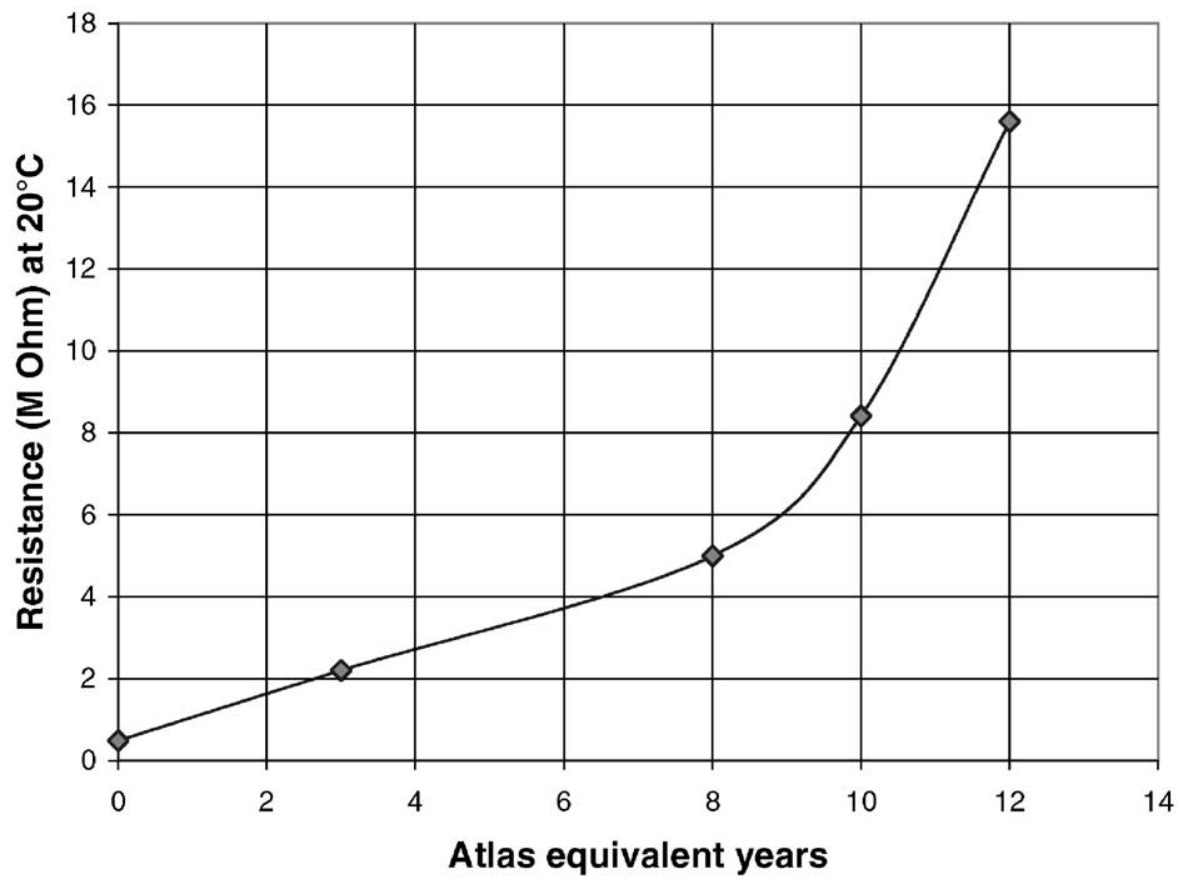

Fig. 5. Plate resistance vs. ageing measured in AY.

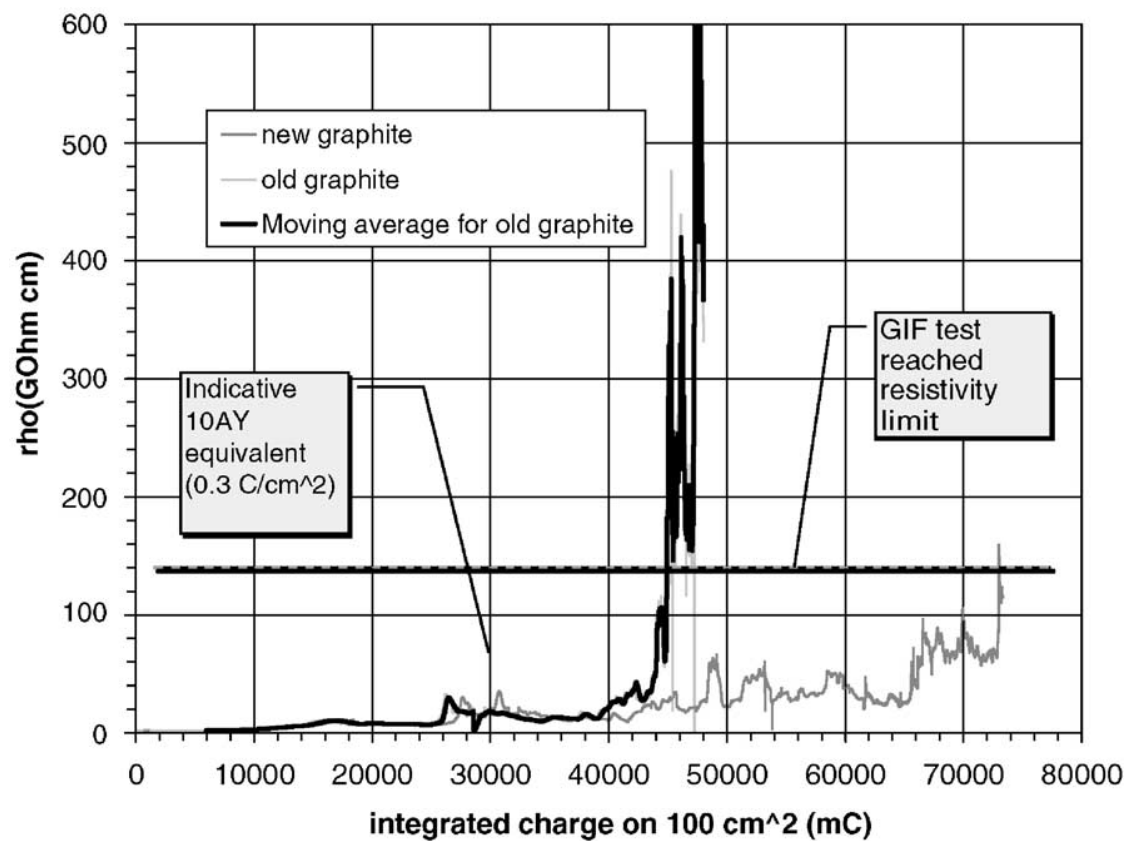

Fig. 6. Plate resistivity vs. integrated charge for the old and the new graphite. 
In order to estimate the contribution of this damaging to the total plate resistance, a further test was conducted in parallel on an identical plate, coated with an improved graphite, characterized by a larger amount of graphite per unit surface, while keeping the same surface resistivity. The result, shown by the pale curve, suggests that the new graphite is more robust against this kind of damage since the dashed line limit is reached at an integrated charge of $\sim 0.8 \mathrm{C} \mathrm{cm}^{-2}$.

\section{Conclusions}

The ATLAS module " 0 " shows a residual rate capability of $\sim 300 \mathrm{~Hz} \mathrm{~cm}^{-2}$ after $12 \mathrm{AY}$, that is well above the requirements. The cause of most of the rate capability decrease is identified with the damage of the anodic graphite layer, due to the current flowing through the Bakelite plate. A strategy for improving the detector performances is proposed and tested. The results indicate that at least a factor of 2 in the detector lifetime is gained.

\section{References}

[1] ATLAS Muon Spectrometer Technical Design Report CERN/LHCC/97-22 (Chapter 8).

[2] P. Camarri, et al., Nucl. Instr. and Meth. A 414 (1998) 317.

[3] G. Aielli, et al., Nucl. Instr. and Meth. A 456 (2000) 77.

[4] G. Aielli, et al., Nucl. Instr. and Meth. A 414 (1998) 82.

[5] S. Agosteo, et al., A facility for the test of large area muon chambers at high rate, CERN-EP-2000-031, February 16, 2000.

[6] G. Aielli, Master Thesis, October 30, 1997. 